\title{
Desempenho escolar de crianças com Distúrbio Específico de Linguagem: relações com habilidades metafonológicas e memória de curto prazo
}

\author{
School performance of children with Specific Language Impairment: \\ relations with metaphonological skills and short-term memory
}

\author{
Ana Paola Nicolielo ${ }^{\text {, Graciela Barbosa Fernandes }}{ }^{2}$, Vera Lúcia Garcia ${ }^{3}$, Simone Rocha de Vascocellos Hage
}

\section{RESUMO}

\begin{abstract}
Objetivo: Descrever o desempenho de crianças com Distúrbio Específico de Linguagem (DEL) em provas de leitura, escrita, aritmética, consciência fonológica e memória seqüencial auditiva, assim como, verificar se há associação positiva entre as provas que avaliam a aprendizagem escolar e as que avaliam o processamento da informação. Métodos: Vinte sujeitos com diagnóstico de DEL, com idades entre 7 e 12 anos, foram submetidos ao Teste de Desempenho Escolar (TDE) e a duas provas, que avaliam o processamento da informação (Perfil de Habilidades Fonológicas e Subteste de Memória Seqüencial Auditiva do Teste de Illinois de Habilidades Psicolingüísticas - ITPA). Resultados: A maioria apresentou alteração em todas as provas realizadas. As associações entre o desempenho do grupo nas diferentes provas demonstram que a habilidade metafonológica apresentou associação estatisticamente significante com as habilidades de leitura $(\mathrm{p}=0,02)$ e escrita $(\mathrm{p}=0,02)$. Por sua vez, a habilidade de memória seqüencial auditiva apresentou associação estatisticamente significante apenas com a habilidade de aritmética ( $\mathrm{p}=0,0003)$. Conclusão: O desempenho escolar, assim como as habilidades de consciência fonológica e memória de curto prazo mostraram-se defasados na maioria dos sujeitos avaliados, havendo associação positiva entre: a prova de memória de curto prazo e a prova de aritmética; a prova de consciência fonológica e as provas de leitura e escrita. Neste contexto, reforça-se aqui a utilização de programas de intervenção baseados em Modelos Psicolingüísticos, que sugerem o uso de estratégias individuais para o desenvolvimento das habilidades metafonológicas.
\end{abstract}

Descritores: Linguagem infantil; Aprendizagem; Leitura, Escrita; Avaliação de desempenho

\section{INTRODUÇÃO}

A escrita é considerada uma expressão da linguagem oral, realizada por meio de sinais criados pelo próprio homem ${ }^{(1)}$. Contudo, deve-se admitir que a escrita é um sistema de repre-

Trabalho realizado no Departamento de Fonoaudiologia da Faculdade de Odontologia de Bauru da Universidade de São Paulo - USP - Bauru (SP), Brasil, com financiamento concedido pela Fundação de Amparo à Pesquisa do Estado de São Paulo (FAPESP).

(1) Pós-graduanda em Fonoaudiologia do Programa de Pós Graduação da Faculdade de Odontologia de Bauru da Universidade de São Paulo - USP Bauru (SP), Brasil.

(2) Fonoaudióloga clínica do Centro de Assistência Odontológica a Portadores de Necessidades Especiais da Universidade Estadual Paulista Júlio de Mesquita Filho - CAOE/UNESP - Araçatuba (SP), Brasil.

(3) Doutora, Professora do Programa Interação Universidade-Serviço-Comunidade da Faculdade de Medicina de Botucatu da Universidade Estadual Paulista Júlio de Mesquita Filho - UNESP - Botucatu (SP), Brasil.

(4) Doutora, Professora do Departamento de Fonoaudiologia da Faculdade de Odontologia de Bauru da Universidade de São Paulo - USP - Bauru (SP), Brasil.

Endereço para correspondência: Simone Rocha de Vascocellos Hage. Departamento de Fonoaudiologia da Faculdade de Odontologia de Bauru da Universidade de São Paulo. Al. Octávio Pinheiro Brisola, 9/75, Bauru - SP, CEP 17012-901. E-mail: simonehage@usp.br

Recebido em: 26/11/2007; Aceito em: 26/3/2008 sentação da língua e não uma simples transposição gráfica da linguagem oral. A aprendizagem da linguagem escrita nos sistemas alfabéticos, como é o caso da língua portuguesa, depende da capacidade de processar a fala ${ }^{(2)}$; sendo assim, a linguagem oral e linguagem escrita estão intrínsicamente relacionadas ${ }^{(3)}$. Considera-se, então, que este tipo de aprendizado depende, em grande parte, da capacidade do indivíduo em processar a fala. Dessa forma, é esperado que, crianças com prejuízo de linguagem oral apresentem maior probabilidade de manifestar dificuldades de leitura e de escrita, quando comparadas a crianças com desenvolvimento típico de linguagem ${ }^{(4)}$.

Nas pesquisas realizadas neste campo, é claro o envolvimento de alterações de linguagem oral em crianças com dificuldades de aprendizagem ${ }^{(5-6)}$.

As alterações da linguagem oral podem fazer parte das manifestações de diversos quadros que afetam o desenvolvimento infantil. Entretanto, há situações em que essas alterações são específicas, não sendo justificadas por alterações mais globais do desenvolvimento ${ }^{(7)}$. Estes quadros caracterizam o que é denominado de Distúrbio Específico de Linguagem (DEL), que apresenta um amplo espectro, que vai desde um simples atraso até um distúrbio, com alterações de linguagem persis- 
tentes, nas diversas áreas da linguagem (semântica, sintática, pragmática e fonológica), que tendem a afetar a aprendizagem da linguagem escrita ${ }^{(8)}$.

Pesquisas longitudinais com crianças com DEL comprovam que a maioria terá dificuldades no âmbito escolar em graus $\operatorname{variados}^{(9)}$. Na literatura científica, levanta-se a hipótese de que boa parcela das crianças com DEL pode apresentar Distúrbio Específico de Leitura, também denominado de dislexia, em um "continuum" (10).

Muito tem sido discutido a respeito de quais aspectos do processamento da informação lingüística mantêm uma relação direta com o aprendizado da linguagem escrita. Um dos temas que mais tem sido debatido insere-se no âmbito do processamento fonológico (PF), que se refere ao uso da informação fonológica no processamento da linguagem oral e escrita envolvendo, basicamente, três componentes: consciência Fonológica $(\mathrm{CF})$, memória de trabalho fonológica (MTF) e recuperação fonológica ${ }^{(11)}$. Pesquisas sugerem forte relação funcional entre o processamento fonológico e a leitura e a escrita, ou seja, alterações nas habilidades deste processamento podem desencadear alterações no desenvolvimento da linguagem escrita ${ }^{(12)}$.

A relação entre o desenvolvimento de habilidades metalingüísticas, sendo uma delas a consciência fonológica, e a aquisição da linguagem escrita é amplamente descrita na literatura científica. Os resultados de um estudo, em que se propôs a realização de um levantamento de pesquisas realizadas com falantes do Português Brasileiro, acerca dessa relação, no período de 1987 a 2005, mostraram 157 pesquisas, com aumento de frequiência ao longo do período. Estudos sobre consciência fonológica são amplamente dominantes e pesquisas de intervenção surgiram nos últimos anos. Dissertações e teses foram mais numerosas do que artigos publicados, o que sugere a necessidade de maior disseminação do conhecimento produzido $^{(13)}$.

Mas especificamente, déficits na CF têm sido encontrados também nas crianças com DEL, assim como ocorre com crianças com dificuldades específicas na leitura ${ }^{(14)}$. Por outro lado, outros componentes do processamento da linguagem também são apontados como sendo a origem da defasagem lingüística e, como conseqüência, das dificuldades com leitura e com a escrita. Esses componentes envolvem a memória de curto prazo (MCP), incluindo $\mathrm{MTF}^{(15)}$.

Apenas nos últimos 12 anos é que começou o interesse nas pesquisas que envolviam a relação entre a memória de trabalho e o aprendizado das habilidades da língua, bem como o processamento destas habilidades nas crianças com DEL ${ }^{(16-17)}$. Este tipo de memória cumpre um papel ativo no processamento da informação; processando e armazenando as informações de curto prazo é que se mantém o pensamento, a aprendizagem e a comunicação ${ }^{(18)}$. Dessa forma, a MTF permite a codificação da informação fonológica na memória de trabalho, apresentando relação positiva com o grau de severidade do desvio fonológico, permitindo aceitar a idéia de que a memória fonológica está relacionada com a produção da fala ${ }^{(19)}$.

No DEL, pesquisas demonstram que essa capacidade de armazenamento fonológico apresenta-se diminuída, além de apontarem que as tarefas que envolvem a MCP constituem bons marcadores do $\mathrm{DEL}^{(9,20)}$. Conhecer a relação entre a memória fonológica e a habilidade lingüística da criança pode ajudar a compreender melhor as alterações de linguagem ${ }^{(21)}$.

Considerando os estudos que mostram que crianças com DEL no decorrer dos anos apresentam dificuldades no desempenho escolar; que essas dificuldades podem estar relacionadas a limitações na habilidade metafonológica de CF e na MCP; este trabalho teve como objetivos descrever o desempenho de crianças com DEL em provas de leitura, escrita, aritmética, CF e memória seqüencial auditiva (MSA), assim como verificar se há associação positiva entre as provas que avaliam a aprendizagem escolar e as que avaliam o processamento da informação.

\section{MÉTODOS}

Fizeram parte deste estudo 20 sujeitos diagnosticados com DEL, com idades entre 7 e 12 anos (14 do gênero masculino e 6 do gênero feminino). A seleção foi feita a partir da análise dos prontuários da Clínica de Fonoaudiologia da Faculdade de Odontologia de Bauru da Universidade de São Paulo.

Considerando o diagnóstico de DEL, os fatores de inclusão foram: desempenho lingüístico abaixo do esperado para a idade mental e cronológica, considerando-se a expressão e/ou compreensão da linguagem oral; limiares auditivos dentro dos padrões de normalidade; ausência de problemas comportamentais e/ou emocionais; desempenho cognitivo de acordo com a normalidade, ou ainda, discrepância entre o desempenho das habilidades cognitivas verbais e não-verbais ${ }^{(22)}$. Cada sujeito foi avaliado individualmente.

Para verificar a presença ou ausência de prejuízo nas habilidades de escrita, leitura e aritmética foi aplicado o Teste de Desempenho Escolar - TDE ${ }^{(23)}$. O teste é composto por três subtestes: escrita, leitura e aritmética. Os resultados foram analisados considerando como parâmetro o critério escolar.

Para a avaliação do processamento da informação foram aplicadas as seguintes provas:

- Perfil de Habilidades Fonológicas ${ }^{(24)}$, para a verificação da CF, sendo constituído pelos itens que identificam e compõem as habilidades fonológicas (análise, adição, segmentação, subtração, substituição, rimas, rima seqüencial, reversão silábica e imagem articulatória). A análise dos resultados considerou a idade cronológica como parâmetro.

- Subteste Memória Seqüencial Auditiva do Teste de Illinois de Habilidades Psicolingüísticas - ITPA ${ }^{(25)}$, para verificar a MCP. É composto por seqüências de números a serem repetidas pelos sujeitos, quando apresentadas oralmente pelo examinador. Vale ressaltar que esta prova não é a mais adequada para verificar o desempenho da Memória Operacional Fonológica, também denominada de Memória de Trabalho Fonológica. A repetição de pseudopalavras (palavras inventadas) parece ser a forma mais adequada para se avaliar este tipo de habilidade.

Vale ressaltar que todos os procedimentos realizados para aplicação de cada instrumento de avaliação seguiram as normas de aplicação apresentada por seus respectivos autores.

Este estudo teve aprovação do Comitê de Ética em Pesquisa (Processo $n^{\circ}$. 80/2007).

Os resultados foram submetidos a uma análise estatística 
descritiva, com o uso de freqüências absolutas e relativas. Para realização das associações entre o desempenho do grupo nas diferentes provas foi utilizado o teste não paramétrico Qui Quadrado (sendo considerada associação estatisticamente significante quando $\mathrm{p}<5 \%$ ).

\section{RESULTADOS}

No Teste de Desempenho Escolar - $\mathrm{TDE}^{(23)}$, os resultados obtidos demonstraram alteração nas três habilidades testadas para a maioria dos sujeitos (Figuras 1 e 2). Com relação às habilidades de leitura e escrita apenas 1 sujeito (5\%) apresentou desempenho compatível com o critério adotado, que foi o critério de série escolar.

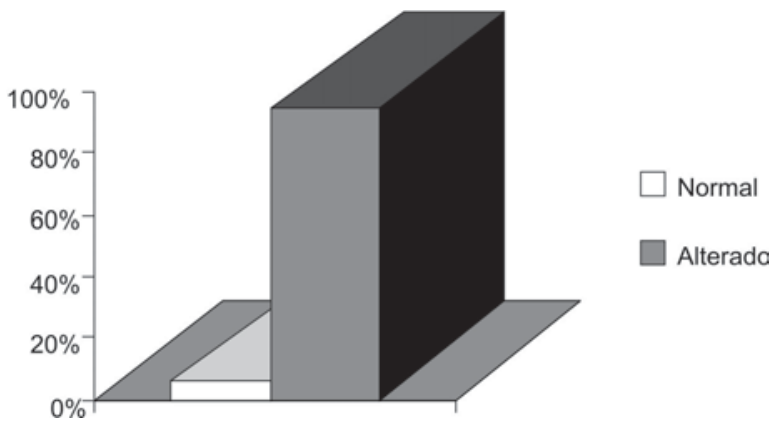

Figura 1. Desempenho dos sujeitos na habilidade de escrita e leitura

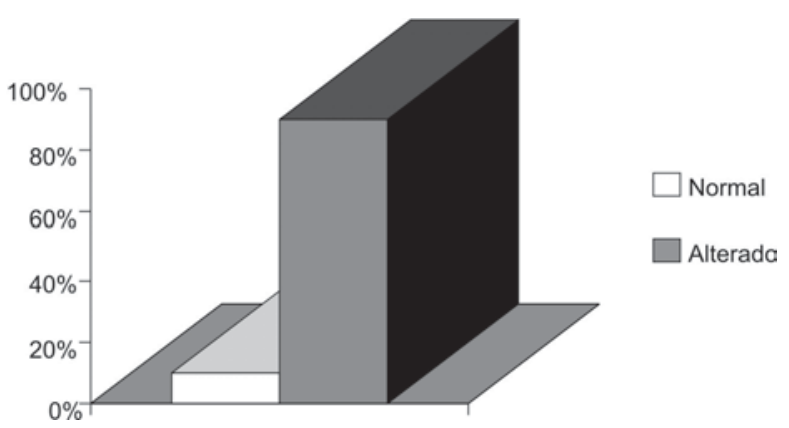

Figura 2. Desempenho dos sujeitos na habilidade de aritmética

Com relação às provas que avaliaram o processamento da informação, no Perfil de Habilidades Fonológicas ${ }^{(24)}, 12$ sujeitos (60\%) apresentaram resultados não mais esperados para a idade cronológica (Figura 3). Quanto ao Subteste Memória Seqüencial Auditiva, do Teste de Illinois de Habilidades

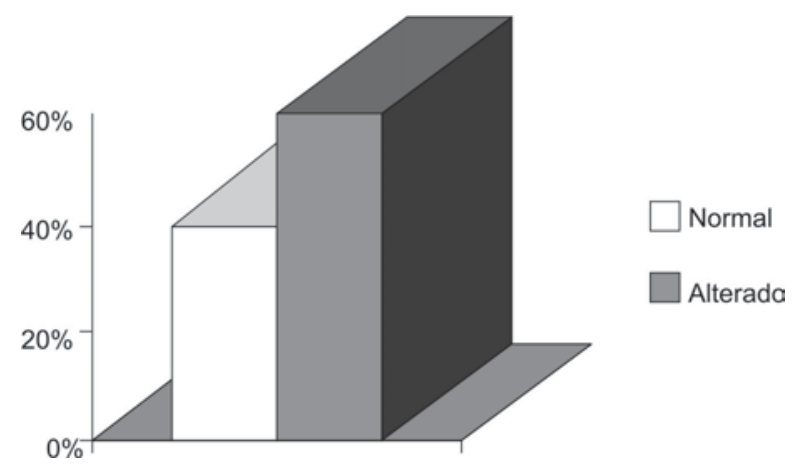

Figura 3. Desempenho dos sujeitos na Habilidade Metafonológica

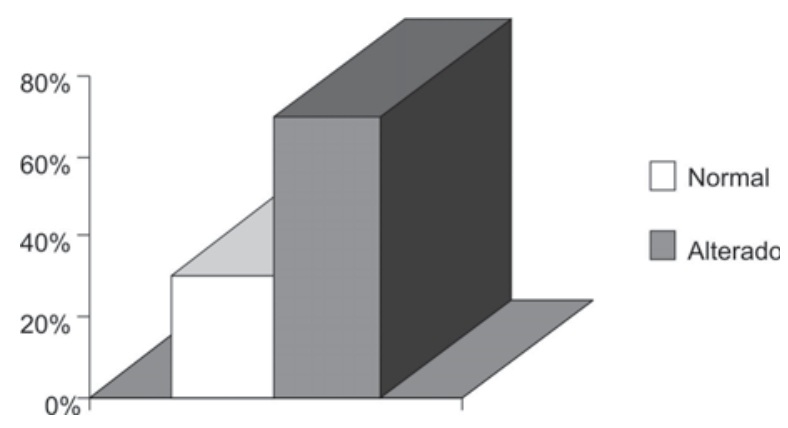

Figura 4. Desempenho dos sujeitos na habilidade de MSA

Psicolingüísticas - ITPA ${ }^{(25)}, 14$ sujeitos (70\%) apresentaram resultados alterados (Figura 4).

Após aplicação do teste não paramétrico Qui Quadrado, para constatar a ocorrência de associações entre as diferentes provas aplicadas, verificou-se que a habilidade metafonológica de CF apresentou associação estatisticamente significante com as habilidades de leitura $(\mathrm{p}=0,02)$ e escrita $(\mathrm{p}=0,02)$. Por sua vez a habilidade de MSA apresentou associação estatisticamente significante apenas com a habilidade de aritmética $(\mathrm{p}=0,0003)$.

\section{DISCUSSÃO}

Após a análise dos resultados constatou-se que a maioria dos sujeitos apresentou alteração em todas as habilidades testadas. A literatura científica apresenta estudos que corroboram com estes achados.

Estudos apontam que, em decorrência das alterações que as crianças com DEL apresentam no processamento fonológico é esperado que, também, ocorram dificuldades na leitura e na escrita ${ }^{(26)}$. Tais alterações incluem déficits na habilidade de consciência fonológica ${ }^{(27)}$, na memória fonológica ${ }^{(28)}$ e no rápido acesso lexical, sendo que este último não foi abordado no presente estudo. Verifica-se, então que os resultados obtidos concordam com a literatura, visto que a maioria dos sujeitos apresentou alteração nas habilidades de consciência fonológica $(60 \%)$ e de MCP (70\%) bem como nas habilidades escolares, em que houve alteração na habilidade de escrita (95\%), de leitura (95\%) e de aritmética (90\%).

Uma pesquisa envolvendo 200 crianças com DEL verificou que apenas $14 \%$ delas apresentavam desempenho acadêmico esperado para série e idade, sem necessidade de apoio extraclasse para as dificuldades com a linguagem oral e escrita ${ }^{(9)}$. Tem sido demonstrado, consistentemente, que as habilidades metalingüísticas, ou seja, a capacidade de refletir sobre a própria língua, são de fundamental importância para aquisição e desenvolvimento da leitura e da escrita ${ }^{(29)}$.

No que tange à associação entre os desempenhos nas diferentes provas, houve associação estaticamente significante entre a prova de consciência fonológica e as provas de leitura $(\mathrm{p}=0,02)$ e escrita $(\mathrm{p}=0,02)$. Por sua vez, a prova de MCP apresentou associação estatisticamente significante com a habilidade de aritmética ( $\mathrm{p}=0,0003)$.

Baixo rendimento escolar e dificuldades com $\mathrm{CF}$ podem estar relacionados, em especial, em crianças com DEL. Isso é compreensível, visto que a $\mathrm{CF}$ corresponde à habilidade para 
identificar e manipular explicitamente as unidades fonológicas da palavra falada, palavra esta que está afetada, quase sempre, por simplificações fonológicas. Conseqüentemente, irá afetar o aprendizado escolar, principalmente no caso da língua portuguesa em que há relação entre o fonema (som) e o grafema (letra).

Déficit de MCP, em especial a MTF, também vem sendo apontado como uma das hipóteses para as dificuldades de linguagem oral e escrita das crianças com DEL. A investigação do desempenho de 20 escolares com DEL, com idades entre 7-11 anos, em testes padronizados de MCP e MTF revelou que a maior parte das crianças apresentou deficiências em MCP $(70 \%)$ e MTF $(95 \%)^{(20)}$. Ressalta-se que estas crianças estavam inseridas em programas de intervenção.

Nosso estudo não apontou uma associação estaticamente significante entre as provas de leitura e escrita e a de MCP. Todavia, a maioria dos sujeitos apresentou defasagem nesta prova de processamento. É provável que a aplicação de provas de memória que utilizasse a repetição de pseudopalavras pudesse revelar uma relação significativa com as provas leitura e escrita, na medida em que esse tipo de prova tem sido descrita como bastante sensível ao funcionamento da MTF, um dos componentes do PF, apontado como sendo a origem da defasagem lingüística das crianças com DEL, e como conseqüência, das suas dificuldades escolares ${ }^{(30)}$.

\section{CONCLUSÃO}

Os resultados permitiram concluir que o desempenho escolar, assim como as habilidades de CF e MCP mostraram-se defasados na maioria das crianças avaliadas, havendo associação positiva entre a prova de MCP e a prova de aritmética, e entre a prova de $\mathrm{CF}$ e as provas de leitura e escrita. Neste contexto, reforça-se aqui a utilização de programas de intervenção baseado em Modelos Psicolingüísticos, que sugerem o uso de estratégias individuais para o desenvolvimento das habilidades metafonológicas.

\begin{abstract}
Purpose: To describe the performance of children with Specific Language Impairment (SLI) in tests of reading, writing, arithmetic, phonological awareness and auditory sequential memory abilities, as well as to verify whether there is a positive association between the tests that evaluate school learning and those that evaluate information processing. Methods: Twenty children with SLI diagnosis and ages between 7 and 12 years, were submitted to the School Performance Test (Teste de Desempenho Escolar-TDE) and two tests that evaluate information processing (Phonological Abilities Profile and the subtest of Auditory Sequential Memory of the Illinois Test of Psycholinguistic Abilities- ITPA). Results: The majority of the subjects presented deficits in all the tests. The analysis of the associations among the group's performance in the different tests showed that metalinguistic skills were significantly associated with the reading $(\mathrm{p}=0.02)$ and writing $(\mathrm{p}=0.02)$ abilities. In turn, the auditory sequential memory ability presented statistically significant association only with arithmetic ability ( $\mathrm{p}=0.0003$ ). Conclusion: The majority of the evaluated children showed deficits in their school performance, as well as in the abilities of phonological awareness and short term memory. It was found positive associations between the short term memory and the arithmetic tests, and between the phonological awareness and the reading and writing tests. In this context, it is emphasized the use of intervention programs based on Psycholinguistic Models, that suggest the use of individual strategies for the development of metalinguistic skills.
\end{abstract}

Keywords: Child language; Learning; Reading; Employee performance appraisal

\section{REFERÊNCIAS}

1. Schiavoni A. Dificuldades de aprendizagem em escrita e percepção de alunos sobre expectativas de professores. [Dissertação Mestrado]. Campinas: Faculdade de Educação da Universidade Estadual de Campinas; 2004.

2. Share L. Phonological recoding and self-teaching: sine qua non of reading acquisition. Cognition. 1995;55(2):151-218; discussion 21926.

3. Salgado C, Capellini SA. Desempenho em leitura e escrita de escolares com transtornos fonológicos. Psicol Esc Educ. 2004;8(2):179-88.

4. American Speech-Language-Hearing Association (ASHA). SpeechLanguage Pathologists' Role in Reading and Writing [Internet]. Rockville: ASHA; c1997-2008. [ cited: 2006 Nov 25] Avaliable from: http://www.asha.org/about/news/tipsheets/SLPs-Role.htm

5. Bird J, Bishop DV, Freeman NH. Phonological awareness and literacy development in children with expressive phonological impairments. J Speech Hear Res. 1995;38(2):446-62
6. França MP, Wolff CL, Moojen S, Rotta NT. Aquisição da linguagem oral: relação e risco para a linguagem escrita. Arq Neuropsiquiatr. 2004;62(2B):469-72.

7. Hall DM, Hill PD. Communication disorders. In: Hall DM, Hill PD, editors. The child with a disability. London: Blackwell; 1996. p.173209.

8. Hage SRV, Guerreiro MM. Distúrbio específico de linguagem: aspectos lingüísticos e neurobiológicos. In: Ferreira LP, Befi-Lopes DM, Limongi SCO, organizadores. Tratado de fonoaudiologia. São Paulo: Editora Roca; 2004. p.977-86.

9. Conti-Ramsden G, Botting N, Simkin Z, Knox E. Follow-up of children attending infant language units: outcomes at 11 years of age. Int J Lang Commun Disord. 2001;36(2):207-19.

10. Conti-Ramsden G. Continuidad académica y educativa en niños con trastorno específico del lenguaje. Rev Chil Fonoaudiol. 2002;3(1):2538. 
11. Wagner RK, Torgesen JK. The nature of phonological processing and its causal role in the acquisition of reading skills. Psychol Bull. 1987;101(2):192-212.

12. Capovilla AGS, Capovilla FC, Suiter I. Processamento cognitivo em crianças com e sem dificuldades de leitura. Psicol Estud. 2004;9(3):44958.

13. Maluf MR, Zanella MS, Pagnez KSMM. Habilidades metalingüísticas e linguagem escrita nas pesquisas brasileiras. Bol Psicol. 2006;56(124):6792.

14. Capellini SA, Poleti FS, Renzo GB, Arruda PD, Pieroni R, Miura RY, et al. Formação de interlocutores para a construção da linguagem escrita: manual de orientação a pais e professores de crianças com dificuldades escolares. Temas Desenvolv. 2000;9(50):33-6.

15. Ratner BN, Gleason BJ, Narasimhan B. Introduction to psycholinguistics: what do language users know? In: Gleason JB, Ratner NB, editors. Psycholinguistics. 2nd ed. Orlando: Harcourt Brace; 1998. p.1-40.

16. Weismer SE. Capacity limitations in working memory: the impact on lexical and morphological learning by children with language impairment. Top Lang Disord. 1996;17(1):33-44.

17. Montgomery JW. Understanding the language difficulties of children with specific language impairments. Does verbal working memory matter? Am J Speech Lang Pathol. 2002;11:77-91.

18. Montgomery JW. Working memory and comprehension in children with specific language impairment: what we know so far. J Commun Disord. 2003;36(3):221-31.

19. Linassi LZ, Keske-Soares M, Mota HB. Habilidades de memória de trabalho e o grau de severidade do desvio fonológico. Pro-Fono. 2005;17(3):383-92.
20. Archibald LM, Gathercole SE. Short-term and working memory in specific language impairment. Int $\mathrm{J}$ Lang Commun Disord. 2006;41(6):675-93.

21. Jeronymo RRF, Galera CA. A relação entre a memória fonológica e habilidade lingüística de crianças de 4 a 9 anos. Pro-Fono. 2000;12(2):55-60.

22. Leonard LB. Children with specific language impairment. Boston: MIT Press; 1997.

23. Stein LM. Teste de desempenho escolar: manual para aplicação e interpretação. São Paulo: Casa do Psicólogo; 1994.

24. Alvarez AMMA, Carvalho IAM, Caetano AL. Perfil de habilidades fonológicas. 2a. ed. São Paulo: Via Lettera; 2004.

25. Bogossian MA, Santos MJ. Teste Illinois de habilidades psicolingüísticas. Rio de Janeiro: EMPSI; 1977.

26. Catts HW, Fey ME, Tomblin JB, Zhang X. A longitudinal investigation of reading outcomes in children with language impairments. J Speech Lang Hear Res. 2002;45(6):1142-57.

27. Nathan L, Stackhouse J, Goulandris N, Snowling MJ. The development of early literacy skills among children with speech difficulties: a test of the "critical age hypothesis". J Speech Lang Hear Res. 2004;47(2):37791.

28. Weismer SE, Evans J, Hesketh LJ. An examination of verbal working memory capacity in children with specific language impairment. J Speech Lang Hear Res. 1999;42(5):1249-60.

29. Cardoso-Martins C. Sensitivity to rhymes, syllables, and phonemes in literacy acquisition in Portuguese. Read Res Q. 1995;30(4):808-28.

30. Baddeley A. Working memory and language: an overview. J Commun Disord. 2003;36(3):189-208. 